\section{Juvenile sterile granulomatous dermatitis (puppy strangle) in Pekingese and German shep- herd puppies}

\section{Mohammad Abbaszadeh Hasiri, Efat Baghaei Moghaddam}

Department of Clinical Studies, School of Veterinary Medicine, Shiraz University, Shiraz, Iran

\begin{abstract}
Juvenile sterile granulomatous dermatitis is an uncommon granulomatous and pustular disorder of the face, pinnae, and submandibular lymph nodes of puppies. A 10-week-old male Pekingese and a 8-week-old female German shepherd presented with submandibular lymphadenomegaly, skin lesions on muzzle and periocular area (Papules, crusts and pustules). The case did not respond to antibiotic therapy. Results of a hemogram, biochemical panel, and urinalysis were normal. Due to skin scraping, cytology examination (impression smear), fungal and bacterial culture and response to therapy puppy strangle (juvenile cellulitis) was diagnosed. The puppies made a full recovery on glucocorticoid therapy. The present case report describes the first report of juvenile sterile granulomatous dermatitis in Iran.
\end{abstract}

\section{Introduction}

Juvenile cellulitis (juvenile pyoderma, puppy strangles, juvenile sterile granulomatous dermatitis and lymphadenitis) is an uncommon granulomatous and pustular disorder of the face, pinnae, and submandibular lymph nodes, usually of puppies.1-3

Puppies can be febrile, depressed, and anorexic. There is an acute swelling of the muzzle, lips, and eyelids. Sterile pustules often develop in the skin of these areas as well as on the inner surface of the pinnae. Otitis externa is common, and pinnae are frequently thickened and edematous. 3,4

After the pustules rupture, small ulcers, draining tracts, seropurulent exudates, or crusts can develop. Submandibular lymphadenopathy occurs and, occasionally, lymph nodes will abscessate and drain. Nodules over the trunk, preputial, and perineal areas due to pyogranulomatous panniculitis, as well as sterile suppurative arthritis, have been reported in a small number of cases. Permanent areas of alopecia and scarring may result if the lesions are extensive. ${ }^{3}$

Diagnosis is based on history, physical examination, and result of skin biopsy of early lesions.4-6

Reported treatments are glucocorticoids and antibiotics, administrated daily until response, then tapered.3,4,6

\section{Case Report \#1}

A 10-week-old, $1 \mathrm{~kg}$ body weight, male Pekingese dog was presented to the clinic of Faculty of Veterinary Medicine, Shiraz University, Iran with clinical signs of depression, anorexia and lesions on muzzle and eyelids. The dog had been treated with Penicillin G (30 mg/kg, IM, q24h for 5 days) by the referring veterinarian, but this treatment did not lead to any significant improvement in the dog's condition. On physical examination, the puppy was pyrexic $\left(39.6^{\circ} \mathrm{C}\right)$, and heart and respiratory rates were within normal range. Submandibular lymphadenomegaly was
Correspondence: Mohammad Abbaszadeh Hasiri, Department of Clinical Studies, School of Veterinary Medicine, Shiraz University, P.O. Box 71345-1731, Shiraz, Iran.

Tel.: +98.71.32288660 - Fax: +98.71 .32286950 .

E-mail: abbaszadeh@shirazu.ac.ir

Key words: Juvenile cellulitis; granulomatous lesions; corticosteroids; dog; Iran.

Contributions: the authors contributed equally.

Conflict of interest: the authors declare no potential conflict of interest.

Received for publication: 26 May 2015

Revision received: 15 July 2015.

Accepted for publication: 15 July 2015.

This work is licensed under a Creative Commons Attribution NonCommercial 3.0 License (CC BYNC 3.0).

(C) Copyright M. Abbaszadeh Hasiri and E.B. Moghaddam, 2015

Licensee PAGEPress srl, Italy

Veterinary Science Development 2015; 5:6037

doi:10.4081/vsd.2015.6037

observed. Papules, crusts and pustular lesions localized on the chin, muzzle and periocular area of this dog were detected (Figure 1A,B). Additionally, otitis externa was present (Figure 1C). Results of a hemogram, biochemical panel, and urinalysis were normal. Impression smears from the exudative lesions from skin or the ear revealed macrophages, with nondegenerate neutrophils. Microorganisms were not seen (Figure 2). No significant parasites, bacteria, and fungi were found on deep and superficial skin scraping and cytology examination. Culture results for bacteria and dermatophytes were negative. Diagnosis of juvenile sterile granulomatous dermatitis was made according to the clinical signs, cytology
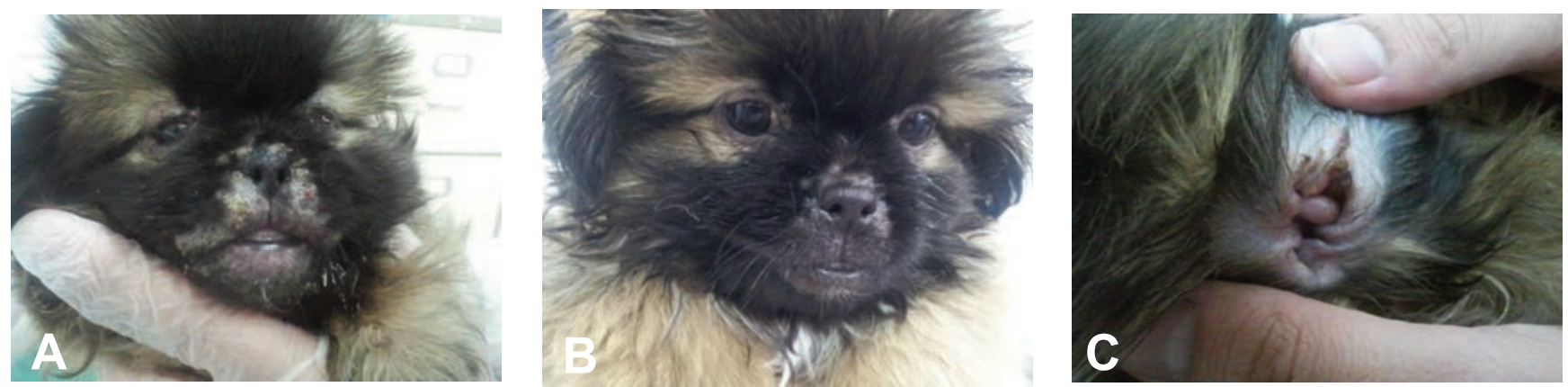

Figure 1. Case \#1: A) lesions in facial region before treatment and B) 7 days after treatment (improvement). C) Otitis externa and purulent exudates in ear of the dog with juvenile cellulitis. 
finding and the results of fungal and bacterial culture. Prednisolone ( $2 \mathrm{mg} / \mathrm{kg}$, po, q24h) for 14 days and continuing for another one week (1 mg/kg, po, 24h) was prescribed. Significant improvement was seen during the first 24-48 hours of treatment (Figure 1A,B). The threemonth follow up showed no regression.

\section{Case Report \#2}

A female 8-week-old German shepherd was referred to clinic with similar signs of case A (Papules, pustules and crusts). In contrast to case 1, there was no otitis or pyrexia. The lesions did not respond to topical treatment with Tetracycline (q12h) and Co-amoxiclav (25 mg/kg q12h). Similar examinations (cytology, skin scraping, fungal and bacterial culture) were made and the same results were obtained. Treatment and signs of improvement were similar (to case 1). Skin lesions before and 7 days after treatment are shown in Figure 3.

\section{Results and Discussion}

The present case report describes the first report of juvenile sterile granulomatous dermatitis in Iran. Juvenile cellulitis is an uncommon acute dermatitis of dogs. Usually, puppies are affected between the ages of 3 weeks and 4 months, and one or several puppies in a litter may have the condition. The etiology and pathogenesis of this condition are unknown. An immunologic abnormality may be involved, as glucocorticoid therapy results in resolution of lesions. There is some evidence for a hereditary factor, as some breeds as well as particular lines within a breed, are predisposed. Predisposed breeds include the golden retriever, dachshund, beagle, pointer and Gordon setter. ${ }^{3-5}$ In the present cases, the dogs were Pekingese and German shepherd puppies and both were non predisposed breeds.

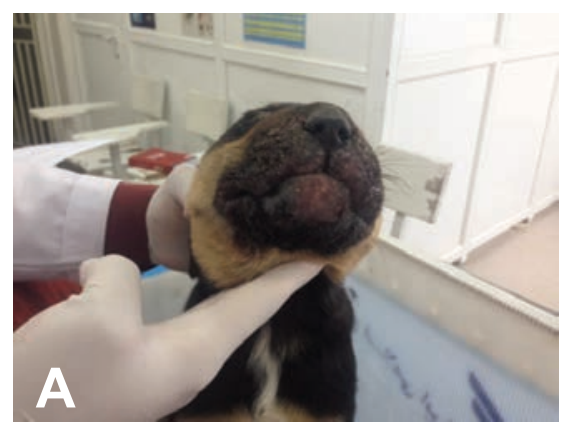

Surprisingly, although the sibling of Pekingese puppy (case 1) was kept elsewhere, he had the similar lesions (he was not referred to the mentioned clinic for further investigation). It could be attributed to the hereditary nature of the disease.

The main differential diagnoses include angioedema, staphylococcal dermatitis, demodicosis, and adverse cutaneous drug reaction. ${ }^{3}$ Due to marked regional lymphadenopathy, systemic illness, impression smear and skin scraping results and no history of medication and vaccination, these possibilities (diagnosis) were ruled out. Besides, response to treatment with no regression revealed no possibility of demodicosis, pyoder- ma or any autoimmune disease (like Pemphigus foliaceus). 4,5 As in these cases, cytologic examination of papulopustular lesions of juvenile cellulitis reveals pyogranulomatous inflammation with no microorganisms and bacterial or fungal cultures are negative. Biopsies of early lesions reveal multiple discrete or confluent granulomas and pyogranulomas consisting of clusters of large epithelioid macrophages with variably sized cores of neutrophils. ${ }^{3}$

These two cases were the only puppy strangles cases in 2014 and among 2100 cases that referred to Shiraz Veterinary School Clinic during this time, the prevalence of this disease is $0.09 \%$.

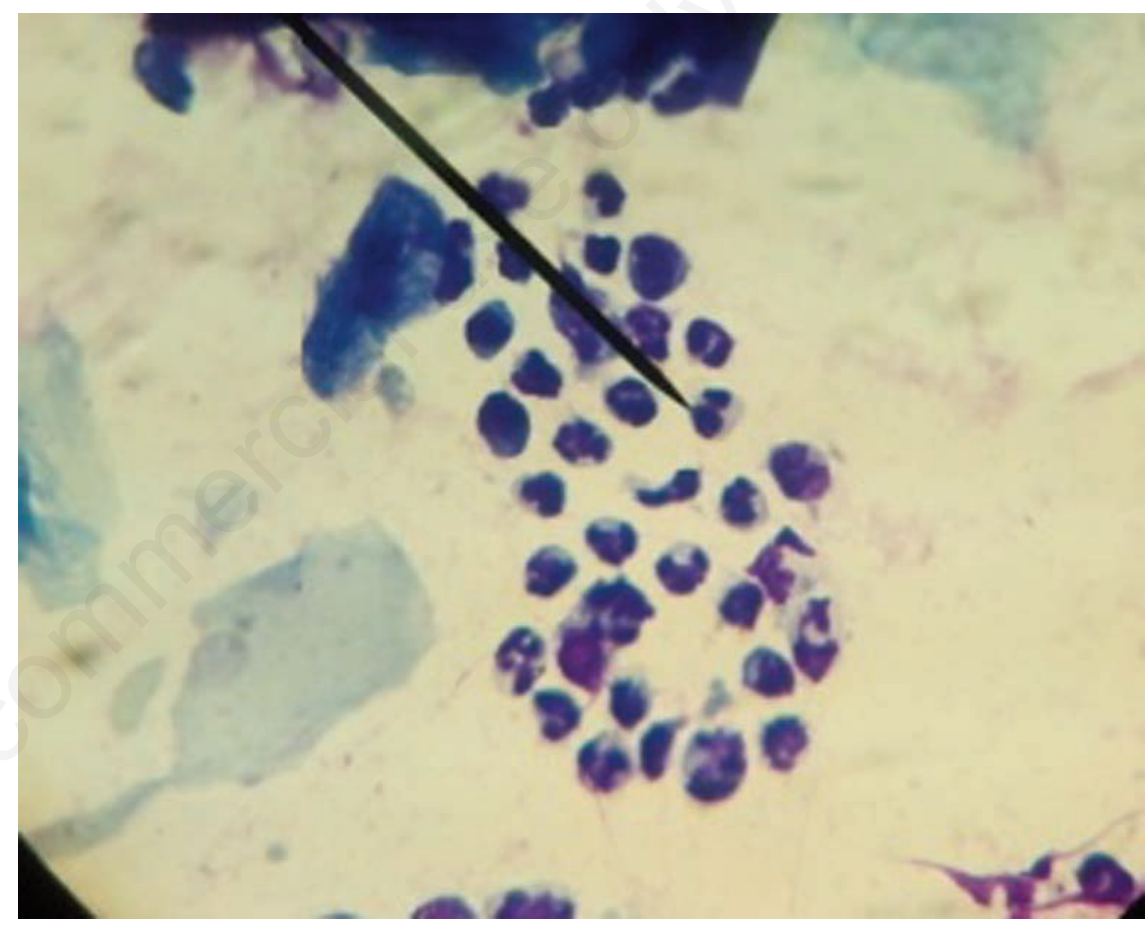

Figure 2. Non-degenerate neutrophils (pointer) in the dog with juvenile cellulitis.
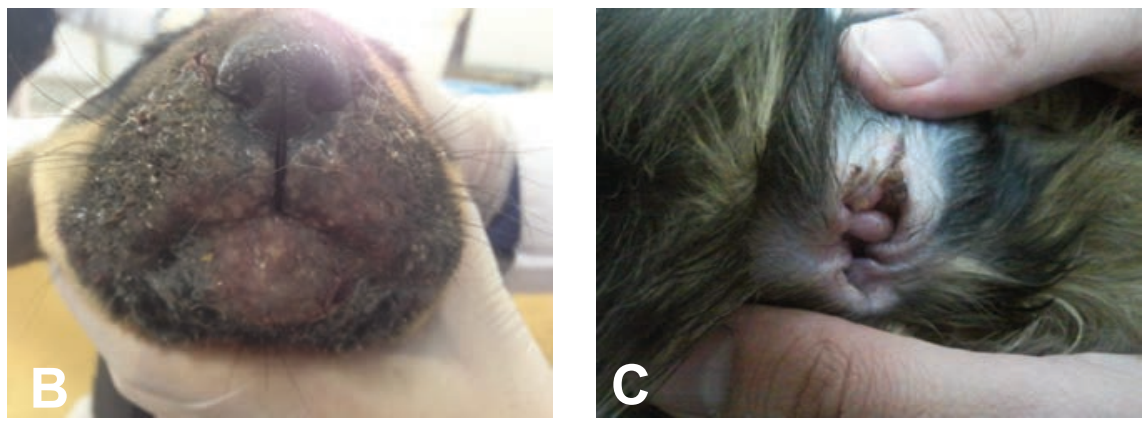

Figure 3. Case \#2, A,B) lesions in facial region before treatment and C) 7 days after treat 


\section{Conclusions}

The juvenile cellulitis could be fatal if not treated. The prognosis is good and recovery without scarring is possible if early treatment is undertaken. ${ }^{5}$ Prednisolone (with or without cyclosporine), oral dexamethasone and topical therapy, especially wet soaks with aluminum acetate or magnesium sulfate have been mentioned in treatment of this disease. ${ }^{3-5}$ The present cases were successfully treated with oral prednisolone.

When puppies are first presented with what appears to be staphylococcal pyoderma, juve- nile cellulitis, a relatively rare condition, may not be considered. However, it is important that the possibility of juvenile cellulitis be explored early, ${ }^{1,6}$ allowing for initiation of glucocorticoid therapy, which is contraindicated for treatment of bacterial pyoderma. $1,3,6$

\section{References}

1. Mason IS, Jones J. Juvenile cellulitis in Gordon setters. Vet Rec 1989;124:642.

2. Reinmann KA, Evans MG, Chalofoux LVS, et al. Clinicopathologic char-acterization of canine juvenile cellulitis. Vet Pathol 1989;26:499-504.

3. Miller WH, Griffin CE, Campbell KL. Muller and Kirk's small animal dermatology. 7th ed. St. Louis, Mosby Elsevier; 2013. pp 709710.

4. McKeever PJ, Nuttal T, Harvey RG. A colour handbook of skin diseases of the dog and cat. 2nd ed. London: Manson Publishing Ltd; 2009. pp 159-160.

5. Paterson S. Manual of skin diseases of the dog and cat. 2nd. Oxford: Blackwell Publishing; 2008. pp 296-297.

6. Hutchings SM. Juvenile cellulitis in a puppy. Can Vet J 2003;44:418-9. 